\title{
THE DENSITY FUNCTIONAL THEORY (DFT) AND DFT+U STUDY OF THE EFFECT OF THE ON-SITE COULOMB REPULSION PARAMETER U ON THE STRUCTURAL AND MAGNETIC PROPERTIES OF $\mathrm{CeO}_{2}$ NANOPARTICLES
}

\section{ALAMGIR KABIR, SADIQ SHAHRIYAR NISHAT AND SUMAYA RAHMAN}

Department of Physics, University of Dhaka, Dhaka 1000, Bangladesh

*Corresponding author e-mail: alamgir.kabir@du.ac.bd

Received on 21.04.2020, Revised received on 14.06.2020, Accepted for publication on 18.06.2020

DOI: https://doi.org/10.3329/bjphy.v27i1.49725

\begin{abstract}
The ab initio density functional theory (DFT) along with DFT+U method was adopted to simulate the bulk and few nanoparticles of Ceria. The nanoparticles studied with different shapes and compositions were $\mathrm{Ce}_{85} \mathrm{O}_{160}$, $\mathrm{Ce}_{44} \mathrm{O}_{80}, \mathrm{Ce}_{19} \mathrm{O}_{32}, \mathrm{Ce}_{13} \mathrm{O}_{24}, \mathrm{Ce}_{13} \mathrm{O}_{32}$. The electronic configuration of the bulk and the nanoparticles were studied very systematically by using DFT and DFT+U approach. It is found that the bulk nonmagnetic $\mathrm{CeO}_{2}$ become magnetic in the nanosized. The magnetic moment of the atoms at different locations in the nanoparticles was studied in detailed and found that there is a significant difference in the magnetization according to the local environment of an atom. We found that the lattice parameter and bandgap obtained with the DFT+U approach give much better agreement with the available experimental value than the DFT approach. To understand the orbital and spin contributions to the magnetic moment, the projected density of states (PDOS) were calculated. We found Ce $\mathrm{d}$ - and f-orbital, and $\mathrm{O}-2 \mathrm{p}$ orbital has the greatest contribution in magnetization and there is ferromagnetic to antiferromagnetic transition of atomic magnetization for some values of $U$. This theoretical study of $\mathrm{CeO}_{2}$ nanoclusters will help to understand the growth dynamics of large nanoparticles and the surface of $\mathrm{CeO}_{2}$ for practical applications.
\end{abstract}

Keywords: DFT; DFT+U; ceria; nanoparticle; magnetism

\section{INTRODUCTION}

Cerium oxide which is simply known as Ceria is one of the most reactive rare-earth metal oxides. It has various applications as catalysis, such as, in fuel cells, it is used as a support for depositing active materials [1] and it has a great ability to take and release oxygen under redox reactions. It has also significant applications in automobile three-way catalyst (TWC), automobile exhaust as oxygen storage, etc.[2] $\mathrm{CeO}_{2}$ is the major component in catalytic converters to reduce harmful emissions from automobile exhausts. [3] In the exhaust of automobiles, $\mathrm{CeO}_{2}$ is used as a purifying $\mathrm{CO}$ exhaust catalyst, it may also be used to reduce hydrocarbon in the emitted gas. [4] In reaction, being inert it directly affects the process of catalysis and due to its reactivity of oxygen transport capability $\left(\mathrm{Ce}^{4+} / \mathrm{Ce}^{3+}\right)$ Ceria has been recognized as an excellent oxygen buffer [5].

Transition metal oxides are strongly correlated material because of their localized d- and f- orbitals where coulomb repulsion energy acts on them on the order of the electronic bandwidth. The electrical, magnetic, optical and other properties of strongly correlated transition metal oxide strongly depends on microscopic properties such as spin, charge, degrees of freedom and orbital magnetic moment of the localized d- and f- electrons, which lead to incredibly rich phenomena [6]. Electron-electron weak interaction can be understood by perturbative methods, Hartree-Fock approximations, Thomas-Fermi approximations, etc. but when the interaction is very strong there needs to develop analytical and computational techniques. 
Nano-structures are of 1-100 nanometers in length in at least one of the dimension exhibit enhanced physical and chemical properties that are not present in the bulk due to change their size, shape, geometry, surface and interface effects or symmetry breaking [7]. Studying their magnetic spins, electrons charges led to discover a new and unique phenomena that cannot be possible in bulk material or with using classical theories.[8] Nonmagnetic bulk materials become magnetic in nanoscale and the magnetic moment increases with the decreases of clusters size.The excellent properties of these materials provide a very promising future and widespread applications in biomedicine[9], catalysis,[10]Nano-electronics[11]. The nanostructure of ceria is found to have large oxidation $\mathrm{CO}$ oxidation than the bulk $\mathrm{CeO}_{2}$ [12]. In the bulk system, where a large number of interacting atoms come into account, quantum mechanics is used to describing the system at microscopic level. As wave function contains a large amount of information of the system, it is not possible to find the exact solution of the Schrödinger equation. In this case, we need to find a suitable approximation specific to the problem and solve the problem analytically.

The ab initio approaches which is based on density functional theory (DFT) (also known as "local density approximation" or simply LDA[13][14] are suitable for studying many bulk and nanosystem, but it fails for strongly correlated Nano-system[15] Generalized Gradient Approximation (GGA) also fails since it cannot explain the exchange-correlation potentials in the strongly correlated system. [16]

In this case, it comes to success with using a parameter " $U$ " which is called Hubbard parameter for local on-site electron-electron repulsion energy. DFT+U method calculates the energy, energy gap, magnetic moment or spins with more accuracy [17][18]. The basic idea behind DFT+U is to treat the strong on-site Coulomb interaction of localized electrons, which is not correctly described by LDA or GGA, with an additional Hubbard-like term. The on-site Coulomb interactions are particularly strong for localized $\mathrm{d}$ and $\mathrm{f}$ electrons but can be also important for $\mathrm{p}$ localized orbitals. The strength of the on-site interactions isusually described by parameters $U$ (on-site Coulomb) and $\mathrm{J}$ (on-site exchange).

This paper focused on the theoretical and analytical study of magnetic properties of Ceria nanoparticles of different sizes and shapes. Among recent studies, Graciani et al.[19] found the electronic and structural properties of Ceria using DFT. The intrinsic defects have been determined by Keating et al.[20] using ab initio molecular dynamics method. Sayle et al.[21]studied the surfaces of Ceria by interatomic potential. The first ab initio investigation was reported by Gennard et al.[22] who used periodic Hartree Fock method. Gagan Ahuja et al.[23] investigated the bandgap of Ceria using LCAO and VASP. Musa Alaydrus et al.[24] studied the contribution of Ce-4f electrons to O vacancies. Loschen et al.[5] investigate binding energies of different octahedral clusters of Ceria using DFT $+\mathrm{U}$ method. They studied some octahedral nano-crystalline clusters using DFT $+\mathrm{U}$ and found it crucial to understand the reactivity of Ceria at the nanoscale.

Since the $\mathrm{CeO}_{2}$ nanocluster is not much been studied, in this paper we focused our attention to thoroughly studied the magnetic properties of $\mathrm{CeO}_{2}$ nanoclusters. We employ both DFT and DFT+U approach in this study and find that DFT+U gives a better approximation than DFT for $\mathrm{CeO}_{2}$ nanoparticles. Five different nanoclusters were formed from the bulk structure of $\mathrm{CeO}_{2}$, these are $\mathrm{Ce}_{85} \mathrm{O}_{160}, \mathrm{Ce}_{44} \mathrm{O}_{80}, \mathrm{Ce}_{19} \mathrm{O}_{32}, \mathrm{Ce}_{13} \mathrm{O}_{24}$, and $\mathrm{Ce}_{13} \mathrm{O}_{32}$. We present a comparative study between the DFT and DFT+U approach here. The valance configuration of Cerium is $5 s^{2} 5 p^{6} 4 f^{1} 5 d^{1} 6 s^{2}$ and the valance configuration of oxygen is $2 s^{2} 2 p^{4}$. For the nanoclusters, the contribution of electrons in different orbitals in magnetization is also studied in detailed. 
DFT+U Approach: The DFT is a very well-established quantum mechanical approach to study the properties of materials. The DFT+U is an extension of DFT which is very suitable for strongly correlated materials. For the strongly localized orbital i.e. if the system has $\mathrm{d}$ or $\mathrm{f}$ orbital the standard DFT underestimates the lattice parameter and bandgap. One needs to have corrections on LDA/GGA energy by Hubbard U parameter, this is known as LDA+U approach as proposed by Dudarev et al. [25]. The total energy in the LDA+U formulation can be written as,

$$
E^{L D A+U}[n]=E^{L D A}[n]+\frac{U}{2} \sum_{i \neq j} n_{i} n_{j}-\frac{U N(N-1)}{2}
$$

One can calculate the value of $U$ from the Hubbard model Hamiltonian

$$
\widehat{H}=\sum_{i i \prime^{\prime}} a_{i \sigma}^{\dagger} t_{i i i^{\prime}} a_{i \prime \sigma}+\sum_{i i^{\prime} j j^{\prime}} U_{i i^{\prime} j j^{\prime}} a_{i \sigma}^{\dagger} a_{i \prime \sigma^{\prime}}^{\dagger} a_{j \prime} a_{i \sigma}
$$

Where $i, i^{\prime} j, j^{\prime}$ represent the different orbitals $\sigma, \sigma^{\prime}$ are different spin orientation, $a, a^{\dagger}$ are the creation annihilation operator.

After doing few more steps one can write the total energy as

$$
E^{L D A+U}=E^{L D A}[n]+\frac{U-J}{2}\left(\sum_{m, \sigma} n_{m, m}^{\sigma}-\sum_{m, m^{\prime} \sigma} n_{m, m^{\prime}}^{\sigma} n_{m^{\prime}, m}^{\sigma}\right)
$$

Where we defined $\mathrm{U}$ and $\mathrm{J}$ as:

$$
\begin{aligned}
& \mathrm{U}=\frac{1}{(2 l+1)^{2}} \sum_{m, m \prime}\left\langle m, m^{\prime}\left|V_{e e}\right| m, m^{\prime}\right\rangle \\
& \mathrm{J}=\frac{1}{2 l(2 l+1)} \sum_{m \neq m^{\prime}}\left\langle m, m^{\prime}\left|V_{e e}\right| m^{\prime}, m\right\rangle
\end{aligned}
$$

The values of $\mathrm{U}$ and $\mathrm{J}$ are obtained by comparing the experimental data with LDA calculation. $\mathrm{DFT}+\mathrm{U}$ approximation gives us a more accurate solution over DFT.

\section{Computational Details}

To visualize the structure and graphics we have used XCrySDen software package [26]. For the bulk calculation, we have used the flourite type structure as shown in Fig. 1. We used DFT as implemented in the VASP package [27] for all the calculations in the ab-initio total-energy process. For the electron-ion interaction, the projector augmented wave method as implemented in the VASP code is used. The conjugate gradient algorithm is used for geometrical optimization, the calculations were optimized if the difference in energy is $10^{-4}$ or less between two successive iterations. The geometric relaxation is achieved by minimizing Hellman-Feynman force by using Davidson algorithm, the force is supposed to be converged if the difference between two successive iterations is $0.01 \mathrm{eV} / \AA$. For the bulk calculation $12 \times 12 \times 12$ irreducible gamma centered KPOINTS are used. For electronic exchange-correlation we used GGA pseudopotential with PW91 functional. The energy cutoff of $400 \mathrm{eV}$ for the plane wave basis set is used. For the nanoparticles, a cubic supercell of size $20 \AA$ x $20 \AA$ x $20 \AA$ is used to avoid the interaction between the particle and its periodic images, and the KPOINTS is this case is $1 \times 1 \times 1$. 


\section{RESULT AND DISCUSSION}

\section{$3.1 \mathrm{Bulk} \mathrm{CeO}_{2}$}

We have started our calculations with the bulk $\mathrm{CeO}_{2}$ structure. $\mathrm{CeO}_{2}$ has fluorite structure with space group Fm3m, it consist of a simple cubic oxygen sublattice with the Ce ions occupying alternate cube centers. The relaxed structure is as shown in the Fig. 1(a). Ce ion (represented by silver ball in Fig. 1(a)) is at the center of the tetrahedron whose corner are occupied by $\mathrm{O}$ atoms (represented by red ball in Fig. 1(a)). The relaxation curve is shown in Fig.1(b) with DFT approach, and in Fig. 1(c) with DFT+U approach. The lattice parameter and the bulk modulus is obtained by fitting the relaxation curve with the Birch-Murnaghan equation of states.

(a)

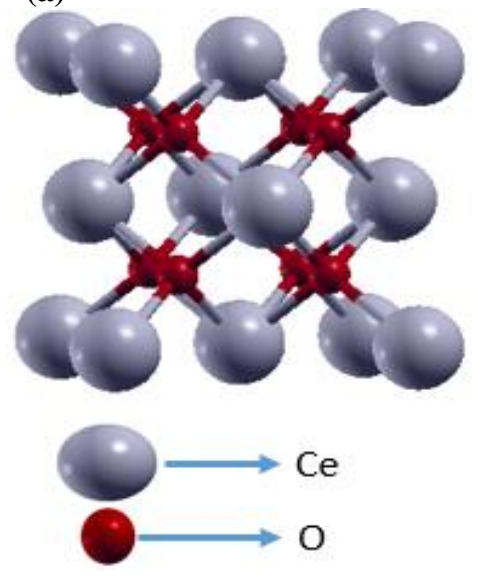

(b)

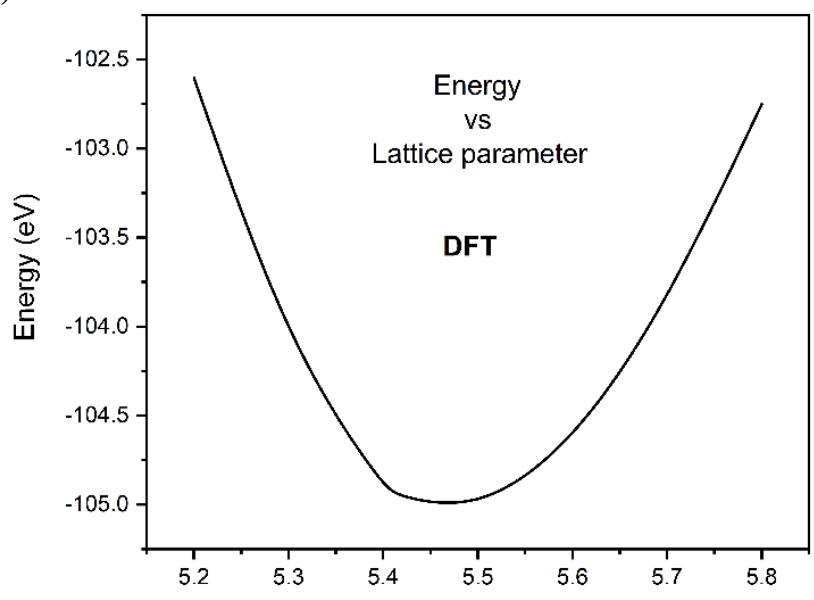

(c)

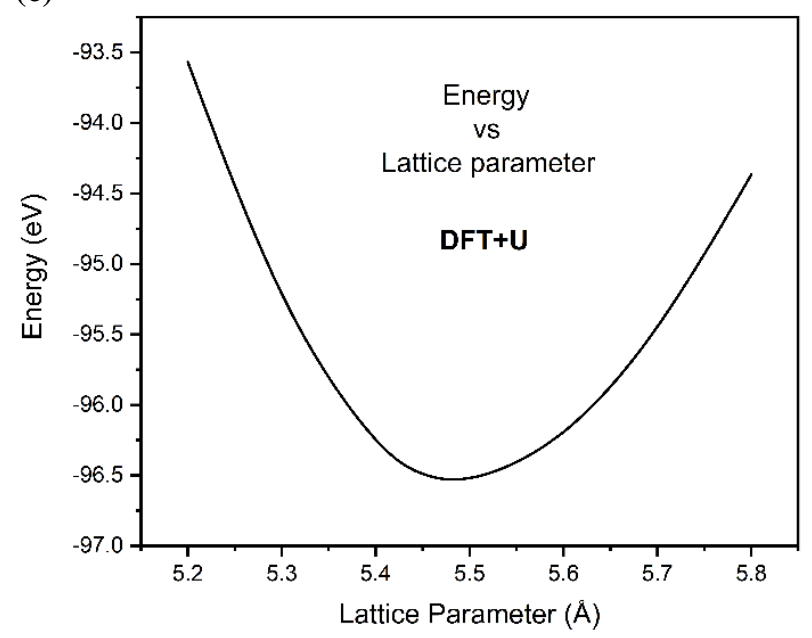

Fig. 1: (a) Bulk structure of $\mathrm{CeO}_{2}$ and Energy vs. Lattice Parameter for $\mathrm{BulkCeO}_{2}$ (b) in DFT approach and (c) in DFT+U approach. 
We find the optimized lattice parameter $\mathrm{a}=5.415 \AA$ for GGA and $\mathrm{a}=5.445 \AA$ for GGA+U. Yong Jiang et al.[28] found the value of the lattice parameter for bulk $\mathrm{CeO}_{2}$ is, a=5.405 $\AA$ which is very close to our findings of GGA. Christoph Loschen et al. [5] found the value of lattice parameter for bulk $\mathrm{CeO}_{2}$ is, $\mathrm{a}=5.4 \AA$. Musa Alaydrus[24] calculated the value $\mathrm{a}=5.429 \AA$ where they found $\mathrm{a}=5.411$ $\AA$ experimentally [29]. The value of the lattice parameter and the bulk modulus obtained in different studies are summarize in table 1 with reference.

Table 1: The lattice parameter and bulk modulus obtained in this study and reported in the literatures.

\begin{tabular}{cccc}
\hline Method & Lattice parameter & Bulk ModulusGPa & Reference \\
\hline GGA & 5.415 & 173.1 & This Study \\
GGA+U & 5.445 & 184.23 & This Study \\
GGA & 5.468 & 172 & {$[30]$} \\
GGA+U & 5.494 & 181 & {$[30]$} \\
GGA & 5.463 & 181 & {$[31]$} \\
GGA+U & 5.494 & & {$[20]$} \\
GGA+U & 5.49 & 180 & {$[32]$} \\
LDA+U & 5.405 & 210.2 & {$[28]$} \\
GGA & 5.429 & & {$[24]$} \\
Experiment & 5.411 & 220 & {$[29][33]$} \\
\hline
\end{tabular}

The distance between two neighboring Ce atoms is $3.71 \AA$ and the Ce-O bond length is $2.50 \AA$ which are in agreement with the value reported in the loschen et al. [5]

The energy band of bulk $\mathrm{CeO}_{2}$ is computed and presented in the Fig. 2 (a,b) by both in DFT and $\mathrm{DFT}+\mathrm{U}$ for $\mathrm{U}=7 \mathrm{eV}$ and $\mathrm{J}=0.7 \mathrm{eV}$. The bandgap indicates in the Fig.3(a) is $1.94 \mathrm{eV}$ with the conduction band minima is at $1.7168 \mathrm{eV}$ and valance band maxima is at $-0.2232 \mathrm{eV}$. For the DFT+U

(a)

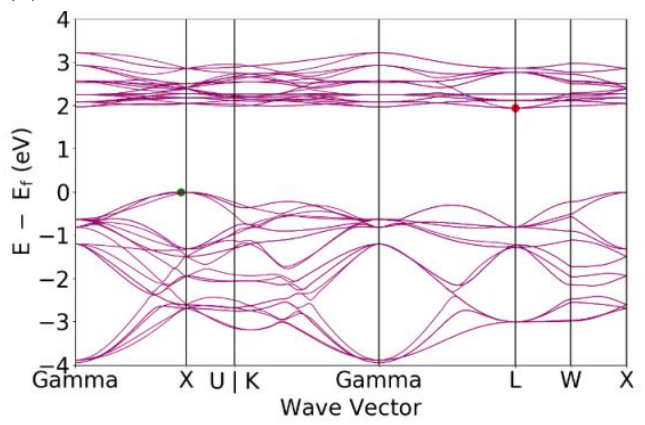

(b)

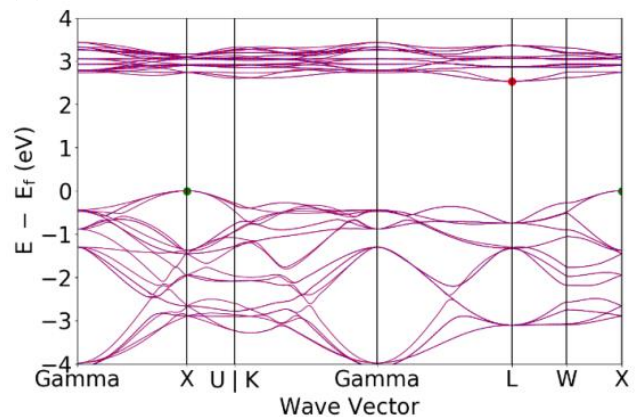

Fig. 2. The band structure of bulk $\mathrm{CeO}$, by using (a) DFT and (b) DFT+U approach. $0 \mathrm{eV}$ indicate the Fermi energy of the system.

Case the minima of the conduction band move upward than DFT and it is found at $2.3116 \mathrm{eV}$, and conduction band maxima remain almost at the same position $(-0.2214 \mathrm{eV})$ as in DFT. The bandgap found in DFT+U is $2.53 \mathrm{eV}$. This bandgap is in good agreement with the experimental bandgap found between $4 \mathrm{f}$ orbital of $\mathrm{Ce}$ and $2 \mathrm{p}$ orbital of $\mathrm{O}$ atoms as reported in [34]. 
To clarify the orbital contribution of the $\mathrm{CeO}_{2}$ in the band structure, the orbital projected density of states of $\mathrm{Ce}$ and $\mathrm{O}$ atom in the $\mathrm{CeO}_{2}$ are shown in Fig. 3(a,d). In the Fig. 3(a) and Fig.4(b) we present PDOS of $4 \mathrm{f}$ orbital of $\mathrm{Ce}$ atom and $2 \mathrm{p}$ orbital of $\mathrm{O}$ atom. We see that the highest occupied state for the spin up or down states of the valance band is composed of the $2 p$ orbital of $\mathrm{O}$ atom for both in DFT and in DFT+U case. The lowest energy unoccupied states of the $\mathrm{CeO}_{2}$ is comprised of the $4 \mathrm{f}$ states of the Ce atoms as indicated by Fig. 3(a \&b). There is no occupancy at the fermi level and the gap between Ce-4f and $\mathrm{O}-2 \mathrm{p}$ is $2.53 \mathrm{eV}$. This is the electronic bandgap of $\mathrm{CeO}_{2}$ as measured by Wuilloud et al. reported in [34].

The PDOS of O-2p state and Ce-5d states are presented in the Fig. 3(c) in DFT and in Fig. 3(d) in $\mathrm{DFT}+\mathrm{U}$ approach. It shows that Ceria has a wide bandgap between O-2p and Ce-5d states, we found the bandgap is around $5 \mathrm{eV}$ in DFT and $5.5 \mathrm{eV}$ in DFT+U approach. This is in good agreement with the previous studies that claim that $\mathrm{CeO}_{2}$ is a wide bandgap semiconductor with the bandgap in the range 5.5 to $6.0 \mathrm{eV}$ [35] The valance band of $\mathrm{CeO}_{2}$ is formed by the $2 \mathrm{p}$ states of $\mathrm{O}$ and the conduction band is formed by the $5 \mathrm{~d}$ states of $\mathrm{Ce}$ atoms. The unoccupied $4 \mathrm{f}$ state lies within the energy gap of the material. [30]

The energy difference between the valance band maxima to the lowest unoccupied $4 \mathrm{f}$ minima is reported earlier to be $3 \mathrm{eV}$ experimentally [36] and $2.35 \mathrm{eV}$ theoretically.[30]
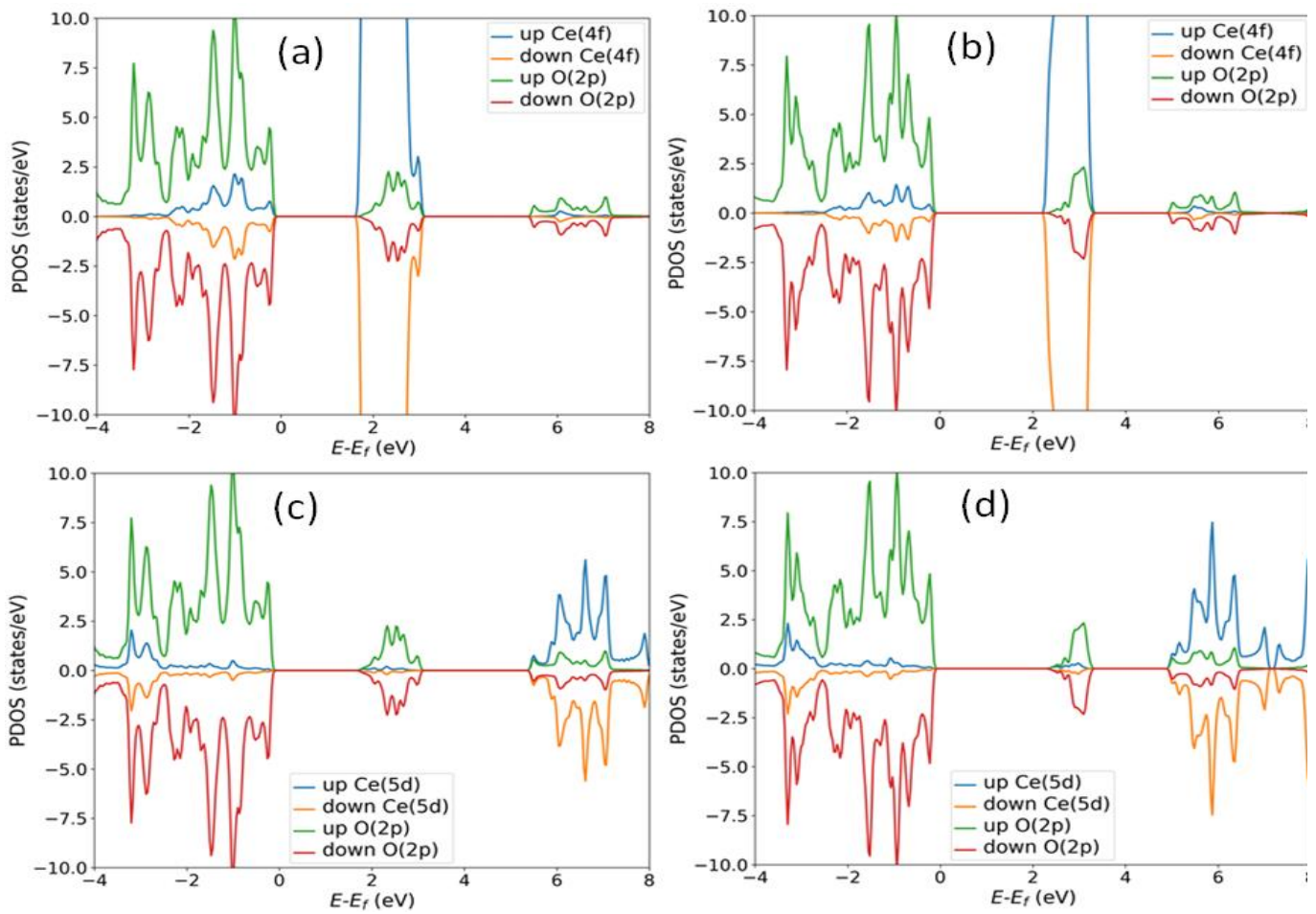

Fig. 3. The spin projected PDOS of Ce-4f and O-2p orbital in (a) DFT and (b) DFT+U; and the PDOS of Ce-5d and O-2p orbital in (c) DFT and (d) DFT+U. 
The Ce-5d to O-2p bandgap of ceria with different approaches is found in the range between 5.14 $\mathrm{eV}$ to $10.75 \mathrm{eV}$, and Ce-4f to $\mathrm{O}-2 \mathrm{p}$ is in the range between $1.26 \mathrm{eV}$ to $7.50 \mathrm{eV}$ as reported in [23]. Our calculated value of bandgap is in the range of previous findings.

\section{$3.2 \mathrm{CeO}_{2}$ Nanoparticles}

The initial structures of $\mathrm{CeO}_{2}$ nanoparticles were formed from the minimum energy bulk $\mathrm{CeO}_{2}$ structure. The relaxed unit cell of the bulk $\mathrm{CeO}_{2}$ (with lattice parameter $\mathrm{a}=5.415 \AA$ ) were multiplied in all the three directions to make a big chunk of bulk material. Then we cut the bulk $\mathrm{CeO}_{2}$ chunk in suitable size and shape to make $\mathrm{CeO}_{2}$ nanoparticles. We have formed five nanoparticles $\mathrm{Ce}_{85} \mathrm{O}_{160}$, $\mathrm{Ce}_{44} \mathrm{O}_{80}, \mathrm{Ce}_{19} \mathrm{O}_{32}, \mathrm{Ce}_{13} \mathrm{O}_{32}$ and $\mathrm{Ce}_{13} \mathrm{O}_{24}$. The nanoparticles were then further relaxed by using DFT \& DFT + U methods. For the relaxation, we have used $1 \times 1 \times 1$ Monkhorst pack KPOINTS grid (the gamma point) sampling in the Brillouin zone, since the unit cell is very large, and $400 \mathrm{eV}$ plane-wave cut-off energy is used. No symmetry constraints were imposed during the structural relaxation. A cubic simulation supercell, with $20 \AA$ distance between the clusters and its periodic images in all three directions, wasimposed in the relaxation calculation of the nanoparticles. The relaxed structure of the nanoparticles are shown in Fig. 4
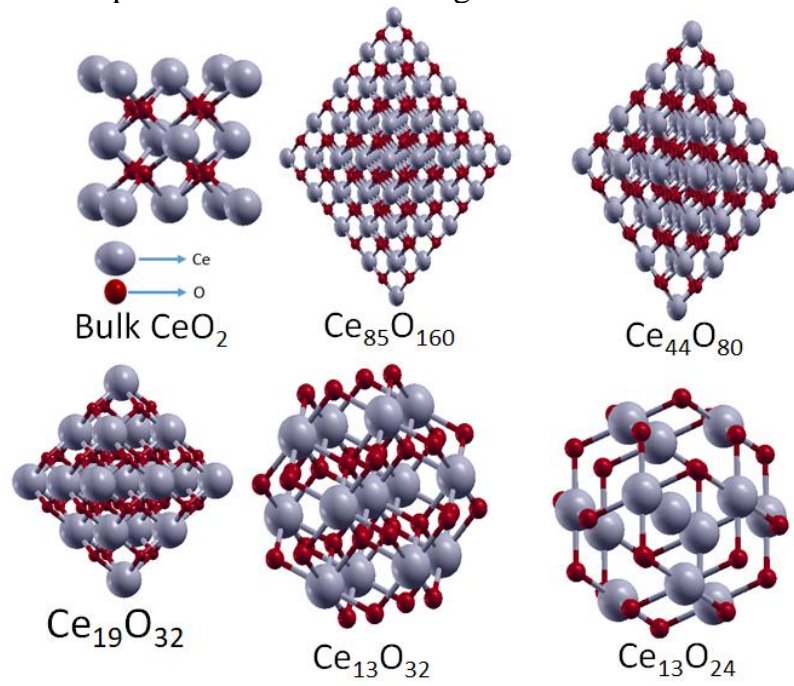

Fig. 4. Different sizes and shapes of $\mathrm{CeO}_{2}$ Nanoparticles studied in this paper. The structural combination is shown below the structures. The silver ball represents the $\mathrm{Ce}$ atom, and the red ball represents the $\mathrm{O}$ atom.

\subsection{1 $\mathrm{Ce}_{85} \mathrm{O}_{160}$ nanoparticle}

We have designed $\mathrm{Ce}_{85} \mathrm{O}_{160}$ Nano-structure by increasing the number of unit cell to $4 \times 4 \times 4$ of the bulk $\mathrm{CeO}_{2}$ and rotate the structure is such a way that the $\mathrm{O}$ terminated surface $\{111\}$ go on the top, we then cut the structure along the $\{111\}$ surface. The structure we obtained is cuboctahedral in shape. The nanoparticle formation mechanism from the bulk structure is justified because it is already shown by the semiempirical model potential that the bulk-like ceria is formed very quickly by agglomerating $\mathrm{CeO}_{2}$ nanoclusters.[21]. The simulated annealing study by Cordatos et al. [37] confirms that the cluster $\left(\mathrm{CeO}_{2}\right) \mathrm{n}$ for $\mathrm{n}=50$ makes bulk like $\{111\}$ surface. The radius of the nanoparticle is $11.6419 \AA$. The corners are occupied with $\mathrm{Ce}$ atom and the central layer is also made with $\mathrm{Ce}$ atom. All the other structures are just derived from the $\mathrm{Ce}_{85} \mathrm{O}_{160}$ structures. We cut the 
atoms from a particular layers to obtain the smaller structures from $\mathrm{Ce}_{85} \mathrm{O}_{160}$. All the structures have central layer formed by $\mathrm{Ce}$ atoms and the corner atoms are also $\mathrm{Ce}$ as in the case of $\mathrm{Ce}_{85} \mathrm{O}_{160}$.

The DOS of the $\mathrm{Ce}_{85} \mathrm{O}_{160}$ Nano-structureare presented in the Fig. 5, both in DFT and DFT+U approach with $\mathrm{U}=6 \mathrm{eV}$. Zero indicates the fermi energy in the graph.

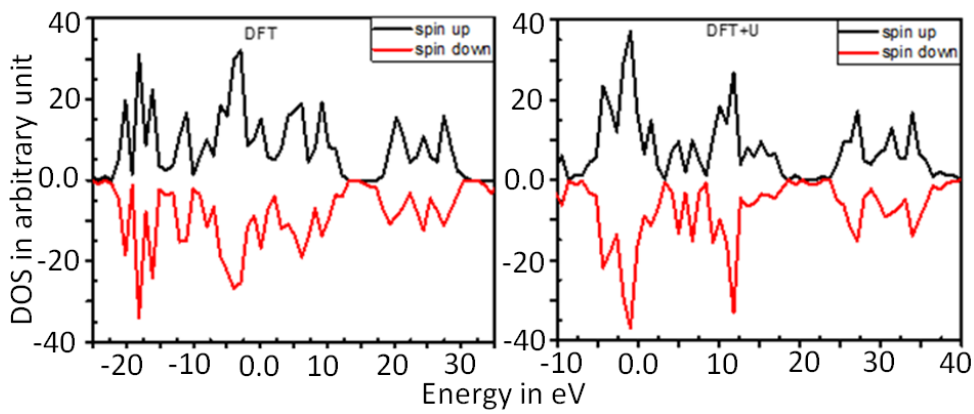

Fig. 5. The total DOS for DFT (left) and DFT+U (right) for $\mathrm{Ce}_{85} \mathrm{O}_{160}$ nanoparticle.

From Fig. 5 we can observe, the peak at $-18 \mathrm{eV}$ in the DFT approach is shifted to $-1 \mathrm{eV}$ and get larger in DFT $+\mathrm{U}$ which leads to a different magnetic moment. The value of magnetic moment in DFT is $1.619 \mu_{B}$ on the other hand, the value of magnetic moment in DFT+U is $-1.873 \mu_{B}$. It is observed that the total magnetic moment of the cluster is positive within the DFT approach, and it became negative within the $\mathrm{DFT}+\mathrm{U}$ approximation. That is the nanoparticle observed a ferromagnetic to anti-ferromagnetic phase transition with increasing the value of $\mathrm{U}$. We explain the transition is due to the fact that some of the center atoms are anti-ferromagnetically coupled with the surface atoms, within the DFT+U approximation the coupling becomes more prominent and the center atoms dominate over the surface atoms, and the nano-particle as a whole became antiferromagnetic. And it is clear from the Fig.6, that the nanoparticle is metallic since it does not show any gap at the fermi level in the DOS.

\subsection{2 $\mathrm{Ce}_{44} \mathrm{O}_{80}$ nanoparticle}

We have designed $\mathrm{Ce}_{44} \mathrm{O}_{80}$ nanostructure, by increasing the bulk unit cell of $\mathrm{CeO}_{2}$ by $3 \times 3 \times 3$ and got a symmetric chunk of bulk material. Consequently, removing atoms side as in the case of $\mathrm{Ce}_{85} \mathrm{O}_{160}$ we get the structure of $\mathrm{Ce}_{44} \mathrm{O}_{80}$ as in Fig. 4. The radius of the nano-particle is $8.7314 \AA$. The corners are occupied with $\mathrm{Ce}$ atom and the central layer is also made with $\mathrm{Ce}$ atom. It has higher magnetization in the surface atoms than in the center atoms. To investigate the magnetization of $\mathrm{Ce}_{44} \mathrm{O}_{80}$ nano-particle we plot DOS as in the Fig. 6. Zero eV is adjusted as the fermi energy of the system.

It is clear from the Fig. 6 that there is no band gap at the fermi energy, that means the nanoparticle is metallic. But there is a gap at higher energy unoccupied state which increases with the value of $\mathrm{U}$, this gap is not important for optical or electronic transition since it is in the unoccupied state. The nanoparticle is magnetic, and the magnetic moment is higher in DFT than in DFT+U case. In $\mathrm{DFT}+\mathrm{U}$ we get the magnetic moment of $2.381 \mu_{B}$, and in the DFT case, the magnetic moment is $4.046 \mu_{B}$. The smaller moment in $\mathrm{DFT}+\mathrm{U}$ is since it locks the itinerant electrons to localized states, so there is less occupancy difference between the up spin and down spin states. 


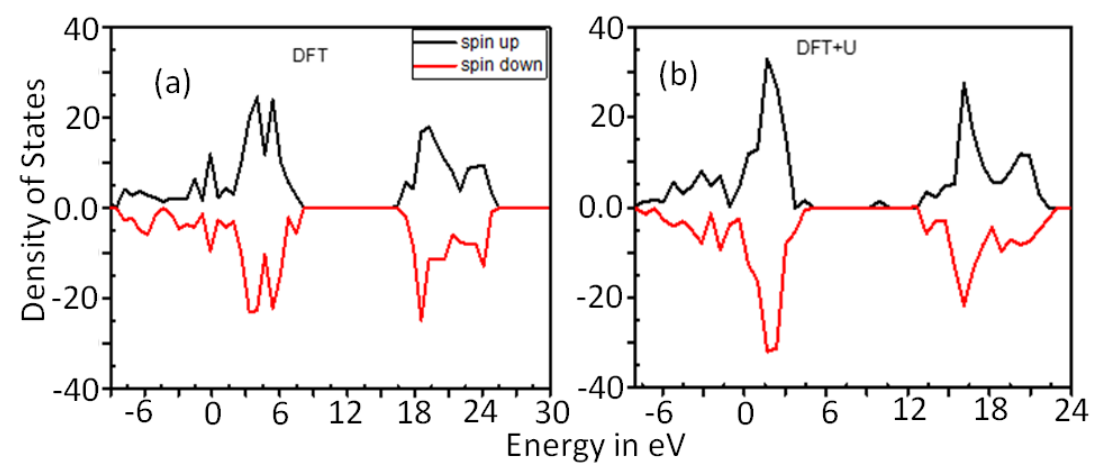

Fig. 6. The total DOS for DFT (a) and DFT+U (b) for $\mathrm{Ce}_{40} \mathrm{O}_{80}$ nanoparticle.

\subsection{3 $\mathrm{Ce}_{19} \mathrm{O}_{32}$ nanoparticle}

The $\mathrm{Ce}_{19} \mathrm{O}_{32}$ nanoparticle is formed by multiplying the optimized bulk $\mathrm{CeO}_{2}$ structure to $2 \times 2 \times 2$ unit cell and cut it along $\{111\}$ direction. We then relax the cluster further by DFT+U approach. The radius of the relaxed nanoparticle is $6.7211 \AA$. The corners are occupied with Ce atom and central layer is also made with Ce atom. The magnetization of the central atoms is higher than the surface atoms in DFT+U calculation, but within the DFT approach we found the opposite, that is the magnetization of inner atoms are smaller than the surface atoms. This nanoparticle is also metallic, means there is no band gap at the fermi energy.

\subsection{4 $\mathrm{Ce}_{13} \mathrm{O}_{32}$ nanoparticle}

The $\mathrm{Ce}_{13} \mathrm{O}_{32}$ nanoparticle is formed from the optimized $\mathrm{Ce}_{19} \mathrm{O}_{32}$ nanoparticle by consequently removing atoms from different sides. The radius of the nanoparticle is $5.8209 \AA$. This structure shows antiferromagnetic properties in both the DFT and DFT+U approach. That is the magnetization of the center atoms are larger than the surface atoms and they have coupled anti-ferromagnetically with the surface atoms. The total DOS of the $\mathrm{Ce}_{13} \mathrm{O}_{32}$ cluster is shown the Fig. 7.

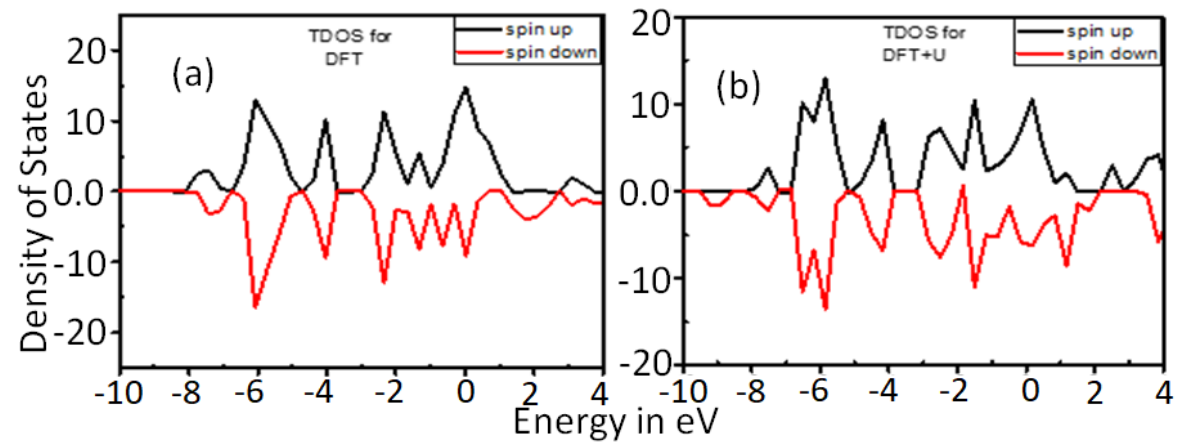

Fig. 7. The total DOS for DFT (a) and DFT+U (b) for $\mathrm{Ce}_{13} \mathrm{O}_{32}$ nanoparticle.

The magnetic moment in DFT approach of the $\mathrm{Ce}_{13} \mathrm{O}_{32}$ nanoparticle $1.238 \mu_{B}$ on the other hand, the magnetic moment in DFT $+\mathrm{U}$ approach is $-4.452 \mu_{B}$ which implies that the cluster is antiferromagnetic in DFT+U approach. We also observed greater splitting of DOS in DFT approach. 


\subsection{5 $\mathrm{Ce}_{13} \mathrm{O}_{24}$ nanoparticle}

The $\mathrm{Ce}_{13} \mathrm{O}_{24}$ is formed by reducing corner atoms from the optimized $\mathrm{Ce}_{19} \mathrm{O}_{32}$ octahedral nanostructure. The radius of the nanoparticle is $5.2208 \AA$. It has higher magnetization in surface atomsbut opposite in direction to the center atom in DFT calculation. On the other hand, it has greater magnetization in center atom butin antiferromagnetically coupled with the surface atoms in the DFT+U calculation. The total DOS of the $\mathrm{Ce}_{13} \mathrm{O}_{24}$ nanoparticle both in DFT and in DFT+U approach is shown in the Fig. 9(a \&b).

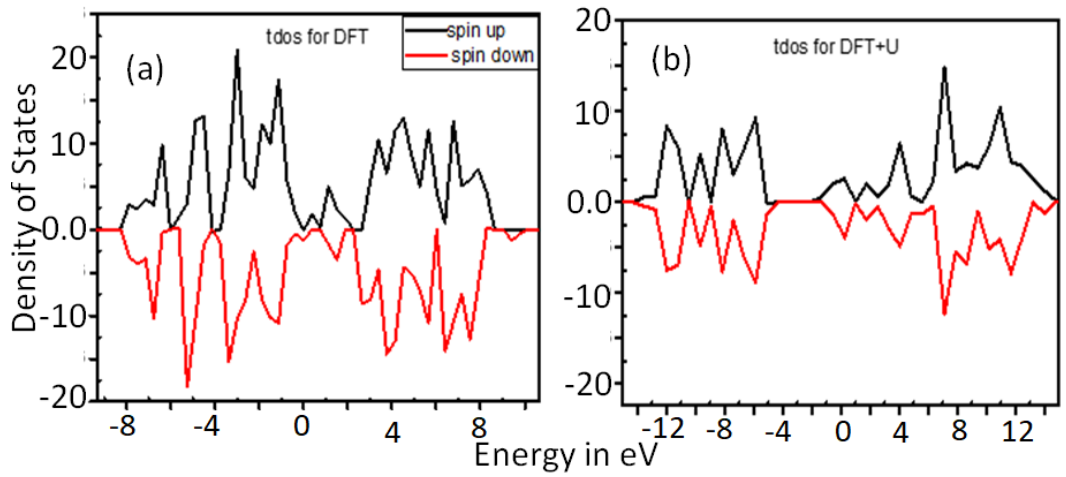

Fig. 8. The total DOS for DFT (a) and DFT+U (b) for $\mathrm{Ce}_{13} \mathrm{O}_{24}$ nanoparticle

From the Fig. 8, we can clearly see that there is no band gap both in DFT and DFT+U approach In DFT approach the peaks at $-13 \mathrm{eV}$ and $-10 \mathrm{eV}$ became dampened in DFT+U approach whereas $\mathrm{DFT}+\mathrm{U}$ plot has peak at $7 \mathrm{eV}$. Which leads to a different spin momentum and so different magnetization than the DFT approach. In DFT approach, the magnetic moment is $1.238 \mu_{B}$ on the other hand the magnetic moment is $-4.452 \mu_{B}$ that indicates anti-feromagneticity in DFT $+\mathrm{U}$ approach.

\section{MAGNETIZATION OF ATMOS AT DIFFERENT POSITIONS IN THE NANOPARTICLES}

To understand the magnetization more detailed we calculated the orbital projected density of states (PDOS) of the surface atoms and the center atoms separately. In Fig. 9, we plot the $d_{z^{2}}$ and $d_{x z}$ orbital of the surface atoms in DFT approach Fig.9(a) and DFT+U approach in Fig.9(c). It is clear that the d-orbital become broadened in DFT+U than in DFT, and the peak height get reduced. 


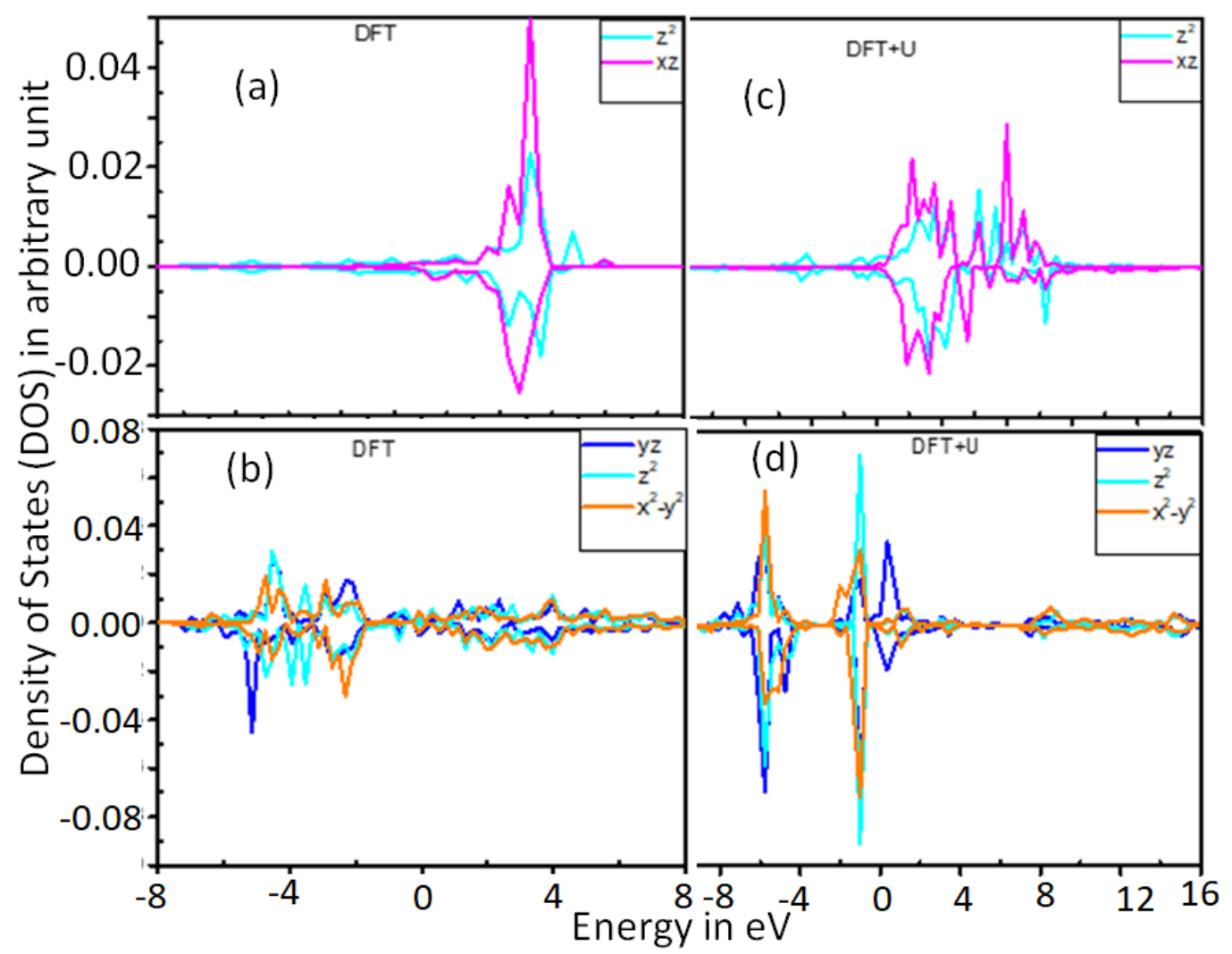

Fig. 9: The DOS for DFT (a) and DFT+U (c) for $d_{z^{2}}$ and $d_{x z}$ orbitals for surface Ce atom. And (b) DFT, (d) $\mathrm{DFT}+\mathrm{U}$ for $\mathrm{Ce}$ atom at the center of nanoparticle.

Similarly, in Fig. 9 (b) and 9 (d) we see that the orbital get broaden and also some extra peak appear in the DFT+U approach which was not present in DFT. The difference in magnetism between surface atoms and center atoms is clear from this figure.

The f-orbital of a $\mathrm{Ce}$ atom which is situated in the center of the $\mathrm{Ce}_{85} \mathrm{O}_{160}$ cluster and a $\mathrm{Ce}$ atom which is situated at the surface of the cluster is presented in Fig. 11, both in DFT and DFT+U approach. Only three orbitals namely $f_{x^{2}}, f_{x\left(y^{2}-z^{2}\right)}$ and $f_{y}\left(z^{2}-x^{2}\right)$ are shown in the Fig. 10, since the other f-orbitals are almost similar in both center and surface atoms. There is significant changes in only this three orbitals present here.

From Fig. 10 we see that $f_{x^{2}}, f_{x\left(y^{2}-z^{2}\right)}$ and $f_{y\left(z^{2}-x^{2}\right)}$ orbitals contribute most to surface Ce atoms f orbital. There is a positive peak at $-25 \mathrm{eV}$ and a large negative peak $-5 \mathrm{eV}$ of $f_{x^{2}}$ orbital in DFT+U which is much smaller in DFT approach. There are also positive peaks of $f_{x\left(y^{2}-z^{2}\right)}$ and $f_{y\left(z^{2}-x^{2}\right)}$ orbitals in DFT+U which are absent it DFT. The states are dampened in DFT approach whereas in $\mathrm{DFT}+\mathrm{U}$ approach energy splitting is greater. This difference in the peak and occupancy of the orbital give the different contribution of magnetization in two different positions of atoms. 


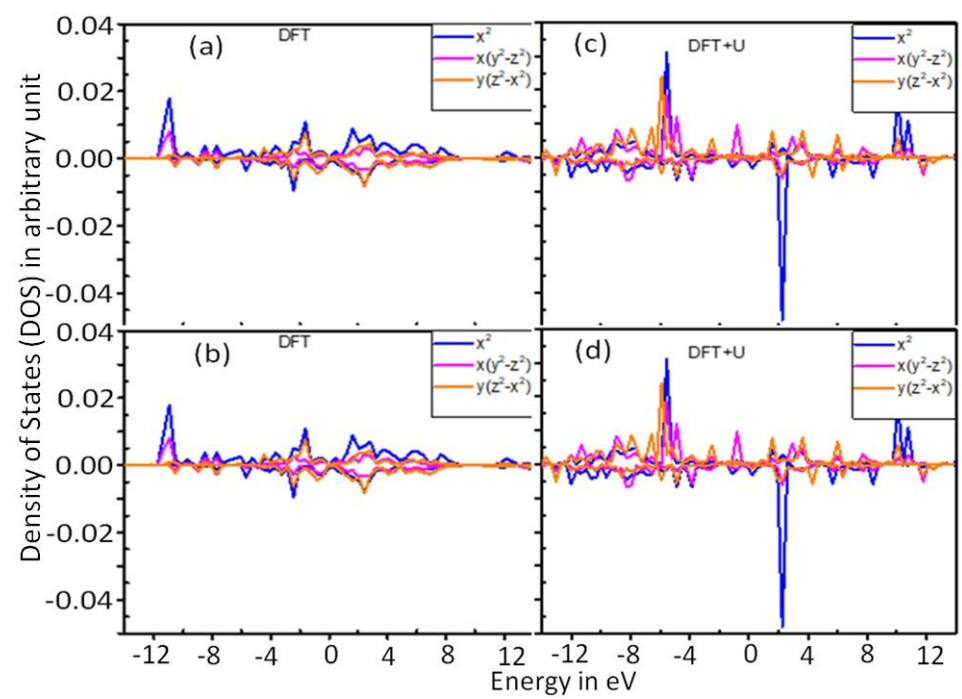

Fig. 10: The DOS for DFT (a) and DFT+U (c) for $f_{x^{2}}, f_{x\left(y^{2}-z^{2}\right)}$ and $f_{y\left(z^{2}-x^{2}\right)}$ orbitals for surface Ce atom. (b) DFT and (d) DFT+U for $f_{x^{2}}, f_{x\left(y^{2}-z^{2}\right)}$ and $f_{y\left(z^{2}-x^{2}\right)}$ orbitals for center atom

The contribution of different orbitals of the $\mathrm{O}$ atoms are also studied very detailed, we plot all the $\mathrm{p}$ orbitals of $\mathrm{O}$ atoms both in the inner and outer positions. We showed the graph in Fig. 11. It is clear that the $p_{x}, p_{y}$, and $p_{z}$ all the orbitals have a significant difference in contribution in DFT and DFT $+U$, also they have significant changes in contribution in the inner and outer atoms.

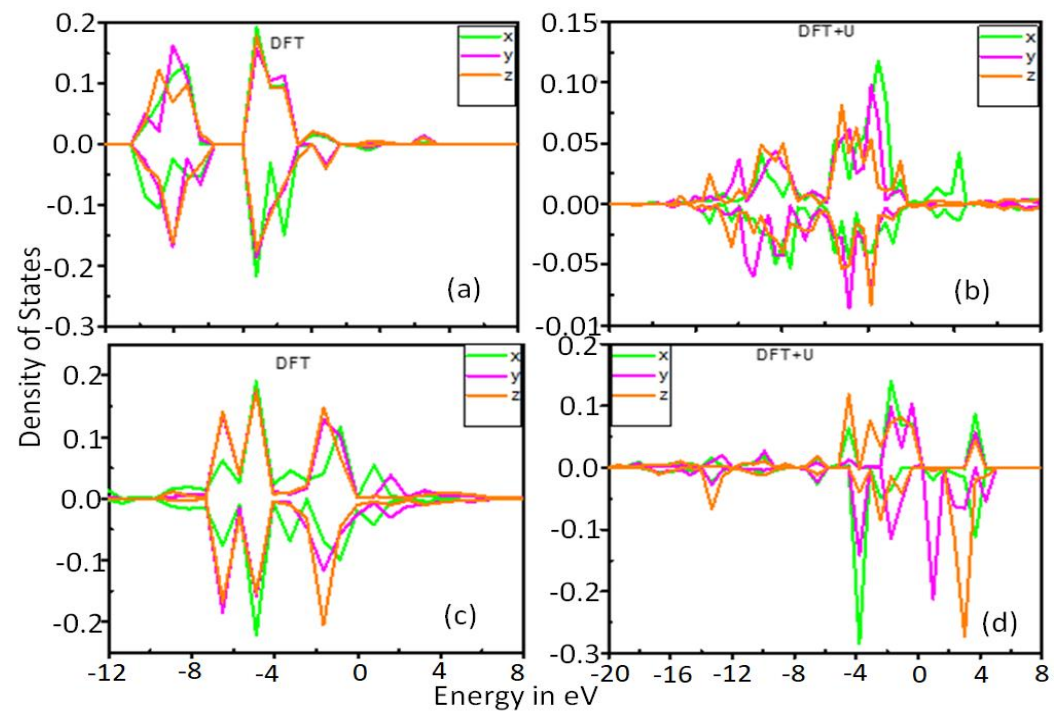

From Fig. 11: the DOS for O atom we observe that, inner layer $2 \mathrm{p}$ orbital is more localized. Energy splitting is higher in DFT+U calculation for center O-2p orbital. Magnetization is much less for O$2 p$ orbitals. 
From Fig. 11(a) and 11(c) it is clear that the PDOS of p-orbitals from the center atoms shifted to the higher energy than the surface atoms. From Fig. 11(b) and 11(c) we see the same phenomenon in $\mathrm{DFT}+\mathrm{U}$, which means the PDOS moved to the higher energy. This shift of the PDOS has a significant contribution of magnetism in the center atoms from the surface atoms.

The atomic contribution of magnetization in the center and surface atoms are presented in Table 2 . It is clear that the value of magnetization does not follow any monotonic relation in magnetization, in some clusters the magnetization of the surface atom is less and in some clusters it is more in surface atoms.

Table 2: The magnetic moment of Ce atoms at different positions in the nanoparticles.

\begin{tabular}{clll}
\hline System & Position & \multicolumn{2}{c}{ Magnetic moment $\left(\mu_{B}\right)$} \\
& & DFT & DFT+U \\
\hline $\mathrm{Ce}_{13} \mathrm{O}_{24}$ & Surface & 0.695 & -0.008 \\
& Center & -0.001 & 0.045 \\
$\mathrm{Ce}_{13} \mathrm{O}_{32}$ & Surface & -0.107 & 0.010 \\
& Center & 0.160 & -0.013 \\
$\mathrm{Ce}_{19} \mathrm{O}_{32}$ & Surface & 0.014 & 0.051 \\
& Center & 0.009 & 0.226 \\
$\mathrm{Ce}_{44} \mathrm{O}_{80}$ & Surface & 0.312 & 0.473 \\
& Middle layer & 0.032 & 0.040 \\
$\mathrm{Ce}_{85} \mathrm{O}_{160}$ & Surface & 0.189 & 0.236 \\
& center & 0.425 & -1.030 \\
\hline
\end{tabular}

From Table 2 we can see that, the magnetic moments are almost same in inner atoms and surface atoms where inner magnetization is slightly larger than surface.

The total magnetization of the cluster in DFT and DFT+U are tabulated in Table 3. In all case the magnetization in DFT $+U$ have larger value in magnetization than in DFT, except for the $\mathrm{Ce}_{44} \mathrm{O}_{80}$ nanoparticle. The large magnetization of $\mathrm{Ce}_{44} \mathrm{O}_{80}$ is due to large number of $\mathrm{Ce}$ atoms on the surface of the $\mathrm{Ce}_{44} \mathrm{O}_{80}$ compared to the others. The change in magnetization that, some of the atoms undergo an antiferromagnetic transition in $\mathrm{DFT}+\mathrm{U}$ case, that is why we have less magnetization in DFT+U.

Table 3: The magnetic moment of the nanoparticles obtained from DFT and DFT+U approach.

\begin{tabular}{|c|c|c|}
\hline \multirow[t]{2}{*}{ Structure } & \multicolumn{2}{|c|}{ Magnetic moment in $\mu_{B}$} \\
\hline & DFT & $\mathrm{DFT}+\mathrm{U}$ \\
\hline $\mathrm{Ce}_{13} \mathrm{O}_{24}$ & 1.238 & -4.452 \\
\hline $\mathrm{Ce}_{13} \mathrm{O}_{32}$ & -0.326 & 0.410 \\
\hline $\mathrm{Ce}_{19} \mathrm{O}_{32}$ & 1.968 & 2.285 \\
\hline $\mathrm{Ce}_{44} \mathrm{O}_{80}$ & 4.046 & 2.381 \\
\hline $\mathrm{Ce}_{85} \mathrm{O}_{160}$ & -1.619 & -1.873 \\
\hline
\end{tabular}

From Table 3 we can see that magnetization has increased in DFT $+U$ calculation for the nanoparticles. The magnetic moment decreased with the increase in size i.e. $\mathrm{Ce}_{13} \mathrm{O}_{24}>\mathrm{Ce}_{13} \mathrm{O}_{32}>\mathrm{Ce}_{19} \mathrm{O}_{32}>\mathrm{Ce}_{44} \mathrm{O}_{80}>\mathrm{Ce}_{85} \mathrm{O}_{160}$ which is a better agreement with Loschen et al. [5] 


\section{CONCLUSIONS}

A systematic analysis of different Cerium and Oxygen terminations of $\mathrm{CeO}_{2}$ was performed using DFT and DFT+U methods to obtain their electronic and magnetic properties. We find that the $\mathrm{DFT}+\mathrm{U}$ method gives the magnetic moments and the bandgaps of Ce atoms with better accuracy than DFT. The magnetic moment decreased with the increase in size which is a better agreement with others. This matches our initial assumption that the strong correlation effect of the partially filled $5 \mathrm{~d}$ and $4 \mathrm{f}$ orbitals electrons cannot be correctly described by the DFT method. Also by comparing the total density (TDOS) with the projected density of state (PDOS) of individual atoms, we see that the inner Ce atoms have significantly more contribution to the total density compared to inner layer atoms this is because the inner atoms of $\mathrm{Ce}-4 f$ orbitals has more neighbor to be hybridized with other orbitals and this hybridization leads to stronger magnetization. Establishing the properties of the surface atoms by doping or changing $\mathrm{O}$ vacancy, we can approximate the characteristics of the corresponding $\mathrm{CeO}_{2}$ nanoparticles.

\section{REFERENCES}

[1] Q. Fu, H. Saltsburg, and M. Flytzani-Stephanopoulos, "Active nonmetallic Au and Pt species on ceriabased water-gas shift catalysts," Science (80-. )., 2003, doi: 10.1126/science.1085721.

[2] M. Anderson, John R., Boudart, Catalysis Science and Technology, Volume 5. Springer, 1984.

[3] M. S. Dresselhaus and I. L. Thomas, “Alternative energy technologies," Nature. 2001, doi: $10.1038 / 35104599$.

[4] Y. F. Yu-Yao and J. T. Kummer, "Low-concentration supported precious metal catalysts prepared by thermal transport," J. Catal., 1987, doi: 10.1016/0021-9517(87)90237-5.

[5] C. Loschen, S. T. Bromley, K. M. Neyman, and F. Illas, "Understanding ceria nanoparticles from firstprinciples calculations," J. Phys. Chem. C, 2007, doi: 10.1021/jp072787m.

[6] A. Asamitsu, Y. Tomioka, H. Kuwahara, and Y. Tokura, "Current switching of resistive states in magnetoresistive manganites," Nature, 1997, doi: 10.1038/40363.

[7] I. M. L. Billas, A. Châtelain, and W. A. De Heer, "Magnetism from the atom to the bulk in iron cobalt, and nickel clusters," Science (80-. )., 1994, doi: 10.1126/science.265.5179.1682.

[8] A. N. Goldstein, C. M. Echer, and A. P. Alivisatos, "Melting in semiconductor nanocrystals," Science (80-. )., 1992, doi: 10.1126/science.256.5062.1425.

[9] R. Elghanian, J. J. Storhoff, R. C. Mucic, R. L. Letsinger, and C. A. Mirkin, "Selective colorimetric detection of polynucleotides based on the distance-dependent optical properties of gold nanoparticles," Science (80-. )., 1997, doi: 10.1126/science.277.5329.1078.

[10] A. T. Bell, "The impact of nanoscience on heterogeneous catalysis," Science. 2003, doi: 10.1126/science. 1083671 .

[11] E. Ozbay, "Plasmonics: Merging photonics and electronics at nanoscale dimensions," Science. 2006, doi: $10.1126 /$ science. 1114849 .

[12] S. Carrettin, P. Concepción, A. Corma, J. M. López Nieto, and V. F. Puntes, "Nanocrystalline $\mathrm{CeO}_{2}$ increases the activity of Au for CO oxidation by two orders of magnitude," Angew. Chemie - Int. Ed., 2004, doi: 10.1002/anie.200353570. 
[13] D. M. Ceperley and B. J. Alder, "Ground state of the electron gas by a stochastic method," Phys. Rev. Lett., 1980, doi: 10.1103/PhysRevLett.45.566.

[14] J. P. Perdew and A. Zunger, "Self-interaction correction to density-functional approximations for manyelectron systems," Phys. Rev. B, 1981, doi: 10.1103/PhysRevB.23.5048.

[15] V. Turkowski, A. Kabir, N. Nayyar, and T. S. Rahman, “A DFT+ DMFT approach for nanosystems,” J. Phys. Condens. Matter, 2010, doi: 10.1088/0953-8984/22/46/462202.

[16] S. G. Ovchinnikov and V. V Val'kov, Hubbard Operators in the Theory of Strongly Correlated Electrons. 2004.

[17] A. I. Liechtenstein, V. I. Anisimov, and J. Zaanen, "Density-functional theory and strong interactions: Orbital ordering in Mott-Hubbard insulators," Phys. Rev. B, 1995, doi: 10.1103/PhysRevB.52.R5467.

[18] F. Cinquini, L. Giordano, G. Pacchioni, A. M. Ferrari, C. Pisani, and C. Roetti, "Electronic structure of $\mathrm{NiO} \mathrm{Ag}(100)$ thin films from DFT+U and hybrid functional DFT approaches," Phys. Rev. B - Condens. Matter Mater. Phys., 2006, doi: 10.1103/PhysRevB.74.165403.

[19] J. Graciani et al., "Comparative study on the performance of hybrid DFT functionals in highly correlated oxides: The case of CeO2 and Ce2O3," J. Chem. Theory Comput., 2011, doi: 10.1021/ct100430q.

[20] P. R. L. Keating, D. O. Scanlon, B. J. Morgan, N. M. Galea, and G. W. Watson, “Analysis of intrinsic defects in CeO 2 using a koopmans-like GGA+ U approach," J. Phys. Chem. C, 2012, doi: $10.1021 /$ jp2080034.

[21] T. X. T. Sayle, S. C. Parker, and C. R. A. Catlow, "The role of oxygen vacancies on ceria surfaces in the oxidation of carbon monoxide,” Surf. Sci., 1994, doi: 10.1016/0039-6028(94)91225-4.

[22] S. Gennard, F. Cora, C. Richard, and A. Catlow, "Comparison of the Bulk and Surface Properties of Ceria and Zirconia by ab Initio Investigations,” J. Phys. Chem. B, 1999, doi: 10.1021/jp9913923.

[23] G. Ahuja, S. Sharma, and G. Arora, "Investigation of electronic structure of $\mathrm{CeO}_{2}$ : first principles calculations," Int. J. Chem. Sci., 14(4), 1907-1917, 2016.

[24] M. Alaydrus, M. Sakaue, and H. Kasai, "A DFT+: U study on the contribution of $4 \mathrm{f}$ electrons to oxygen vacancy formation and migration in Ln-doped CeO2," Phys. Chem. Chem. Phys., 2016, doi: 10.1039/c6cp00637j.

[25] S. L. Dudarev, G. A. Botton, S. Y. Savrasov, C. J. Humphreys, and A. P. Sutton, "Electron-energy-loss spectra and the structural stability of nickel oxide:\&\#8194;\&\#8194;An LSDA+U study," Phys. Rev. B, 1998.

[26] A. Kokalj, "XCrySDen-a new program for displaying crystalline structures and electron densities," $J$. Mol. Graph. Model., 1999, doi: 10.1016/S1093-3263(99)00028-5.

[27] G. Kresse and J. Furthmüller, "Efficient iterative schemes for ab initio total-energy calculations using a plane-wave basis set," Phys. Rev. B - Condens. Matter Mater. Phys., 1996, doi: 10.1103/PhysRevB.54.11169.

[28] Y. Jiang, J. B. Adams, and M. Van Schilfgaarde, "Density-functional calculation of CeO 2 surfaces and prediction of effects of oxygen partial pressure and temperature on stabilities," J. Chem. Phys., 2005, doi: 10.1063/1.1949189.

[29] O. T. Sørensen, "Thermodynamic studies of the phase relationships of nonstoichiometric cerium oxides at higher temperatures," J. Solid State Chem., 1976, doi: 10.1016/0022-4596(76)90099-2.

[30] T. Zacherle, A. Schriever, R. A. De Souza, and M. Martin, "Ab initio analysis of the defect structure of ceria," Phys. Rev. B - Condens. Matter Mater. Phys., 2013, doi: 10.1103/PhysRevB.87.134104. 
[31] M. Nakayama and M. Martin, "First-principles study on defect chemistry and migration of oxide ions in ceria doped with rare-earth cations," Phys. Chem. Chem. Phys., 2009, doi: 10.1039/b900162j.

[32] J. L. F. Da Silva, M. V. Ganduglia-Pirovano, J. Sauer, V. Bayer, and G. Kresse, "Hybrid functionals applied to rare-earth oxides: The example of ceria," Phys. Rev. B - Condens. Matter Mater. Phys., 2007, doi: 10.1103/PhysRevB.75.045121.

[33] L. Gerward, J. Staun Olsen, L. Petit, G. Vaitheeswaran, V. Kanchana, and A. Svane, "Bulk modulus of $\mathrm{CeO}_{2}$ and $\mathrm{PrO}_{2}$-An experimental and theoretical study," J. Alloys Compd., 2005, doi: 10.1016/j.jallcom.2005.04.008.

[34] and Y. B. E. Wuilloud, B. Delley, W. -D. Schneider, "Spectroscopic Evidence for Localized and Extended f-Symmetry States in CeO2," Phys. Rev. Lett., vol. 53, no. 2, p. 202, 1984.

[35] H. Yanagida, R. J. Brook, and F. A. Kröger, "Direct Current-Voltage Characteristics of Calcia Stabilized Zirconia with Porous Platinum Electrodes,” J. Electrochem. Soc., 1970, doi: 10.1149/1.2407585.

[36] D. R. Mullins, S. H. Overbury, and D. R. Huntley, "Electron spectroscopy of single crystal and polycrystalline cerium oxide surfaces," Surf. Sci., 1998, doi: 10.1016/S0039-6028(98)00257-X.

[37] H. Cordatos, D. Ford, and R. J. Gorte, "Simulated annealing study of the structure and reducibility in ceria clusters," J. Phys. Chem., 1996, doi: 10.1021/jp961110o. 